\title{
Holographically recorded gratings on microlenses for a miniaturized spectrometer array
}

\author{
S. Traut \\ H. P. Herzig \\ University of Neuchâtel \\ Institute of Microtechnology \\ Rue Breguet 2 \\ 2000 Neuchâtel, Switzerland
}

\begin{abstract}
We report on the investigation of refractive microlens arrays with diffractive grating surfaces in the context of a microspectrometer array system. The elements fabricated combine the fairly large dimensions of the refractive microlens $(990-\mu \mathrm{m}$ diam, $60-\mu \mathrm{m}$ height) with the submicron features of the diffraction grating ( $1-\mu \mathrm{m}$ grating period) on one transmitting surface. The fabrication process of these elements was studied, as well as their performance with respect to resolution and stray-light suppression. The maximum resolution was $3 \mathrm{~nm}$, and the stray-light suppression $25 \mathrm{~dB}$. We present a concept for a system of miniaturized spectrometer arrays for chemical analysis. (c) 2000 Society of Photo-Optical Instrumentation Engineers. [S0091-3286(00)03001-4]
\end{abstract}

Subject terms: microspectrometer; hybrid element; spincoating; microlens array.

Paper 990067 received Feb. 12, 1999; revised manuscript received June 23, 1999; accepted for publication July 1, 1999.

\section{Introduction}

Today, R\&D efforts in optical demultiplexing systems, as used for chemical analysis or in telecommunications, are directed towards miniaturization and cost reduction. Microoptics provides obvious solutions, particularly for parallel processing with array systems. ${ }^{1}$ It uses standard semiconductor manufacturing technology, offers a variety of optical functions, and is suitable for system integration. ${ }^{2}$ To reduce the size and cost of a system, however, it is desirable to minimize the number of components within the system. This can be achieved by combining different optical functions in one element. In a conventional spectrometer system, the two main optical functions are the focusing and the dispersion. In a hybrid element, these two functions can be combined by fabricating a diffractive surface on either a refractive or a reflective optical element. ${ }^{3-5}$ For planoconvex microlenses, the diffractive elements can be realized on the planar as well as on the curved surface of large arrays. ${ }^{6}$ We have fabricated refractive microlens arrays with the diffraction grating on the curved surface of the array, which combines focusing and dispersion essentially on one transmitting surface. The advantage of such an element lies in the reduction of the total number of optical surfaces within any optical system.

In the following, we present our investigation on the fabrication process of refractive microlens arrays with holographically recorded diffraction gratings (in the following called hybrid elements) and the performance of the element in the context of spectrometer systems. Section 2 deals with the fabrication process and problems associated with it. In Sec. 3 we present the characterization of the elements as microspectrometer elements; Sec. 4 shows raytracing results of different geometries. Finally, in Sec. 5 we summarize the results and present a possible concept of a microspectrometer array system for chemical analysis.

We have chosen holographic recording combined with the melting resist technique because it enables the fabrica- tion of large arrays of elements that have low stray light. Such arrays are difficult to realize with the time-consuming e-beam writing techniques. ${ }^{4}$

\section{Fabrication}

Figure 1 shows a schematic view of the fabrication process for the hybrid elements. Refractive microlens arrays are fabricated by the melting resist technique ${ }^{7}$ in photoresist on a glass substrate. We fabricated arrays of microlenses with different lens diameters and packing densities for the investigation of fabrication characteristics. The lens diameters are 350,610, and $990 \mu \mathrm{m}$ with calculated numerical apertures of $0.34,0.2$, and 0.12 , respectively. The height is 60 $\mu \mathrm{m}$ for all lenses. The sphericity of the lenses was tested with a Twyman-Green interferometer ${ }^{8}$ and PSI software obtained from Prof. Schwider at MIKOS, University of Erlangen, Germany. The lenses fabricated for our experiments exhibited surface deviations from an ideal sphere between $0.2 \lambda$ and $0.4 \lambda$ ( $\mathrm{rms}$ ).

Three different arrays were fabricated for each lens diameter. For the first array the lenses are loosely packed (square packing) with a pitch of $2 \mathrm{~mm}$ between the lenses for all lens diameters. The second array is close-packed (also square packing) with pitches of 355, 618, and 1000 $\mu \mathrm{m}$ for the different lens diameters $(350,610$, and $990 \mu \mathrm{m}$, respectively). The third array has the same pitch between the adjacent lenses as the second one, but is hexagonal close-packed. These lens arrays were then spincoated with photoresist (AZ1518 Hoechst resist, Suess RC8 spincoater). A diffraction grating with a period of $1 \mu \mathrm{m}$ was subsequently recorded by two-beam interference $\left(\mathrm{Kr}^{++}\right.$laser, $\lambda$ $=413 \mathrm{~nm}$ ).

\subsection{Spincoating}

In the fabrication process, the spincoating is a critical processing step. The deposition of the photoresist across the lenses is not isotropic, and the nonhomogeneous distribu- 
(a)

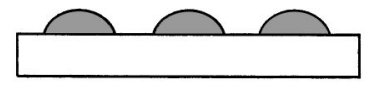

(b)
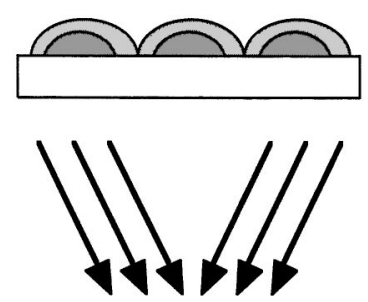

(c)

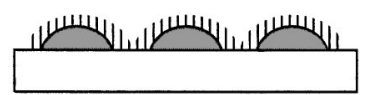

Fig. 1 Fabrication of refractive microlens arrays with a diffraction grating on the curved surface: (a) microlens-array fabrication; (b) photoresist coating of the array; (c) recording of the grating.

tion across the curved surfaces of the lenses changes their optical properties. We can make two general statements about the coating of microlenses. The first statement concerns the smoothing of the edges of the lens-substrate interface. As the profiler measurement in Fig. 2(a) shows, the photoresist is deposited thicker in the corners of the lens- substrate interface than on top of the lens, which results in smooth slopes on the outer diameter of the microlens. Secondly, we learned about the influence of the directionality of the spincoating process on the lens surface. The melting resist technology for fabricating microlenses exhibits inherently a rotational symmetry around the optical axis. However, with the deposition of photoresist on top of a lens array by spincoating, we introduce a processing step, that breaks this symmetry. The result is a non-axiallysymmetric deviation of the surface from an ideal sphere, as shown in Fig. 2(b) for a single lens from a loosely packed array.

Furthermore, we find for close-packed lens arrays that the proximity of the lenses affects the deposition of the photoresist. The mutual influence of single lenses within a close-packed array is demonstrated in Figs. 3(a) and 3(b). Due to the capillary forces, the resist deposition is higher in the proximity of a neighboring lens. The shape of a lens in a square-packed array is therefore deformed to a square. For hexagonal packing, the coating yields a lens with a hexagonal shape.

The sphericity of the lenses is determined with the Twyman-Green interferometer before and after coating. The value measured is the rms value of the deviation from an ideal sphere in $\lambda$.

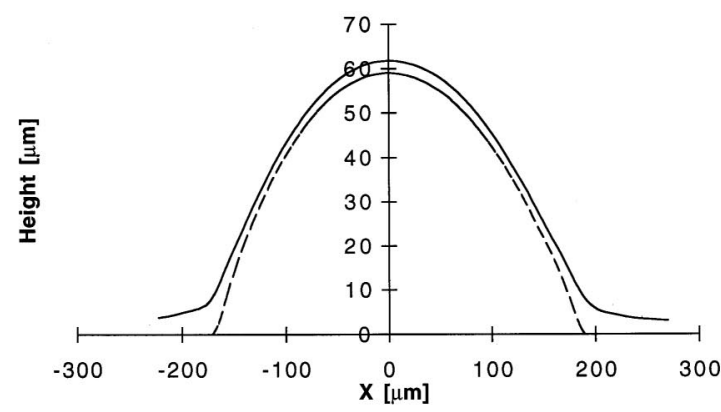

(a)

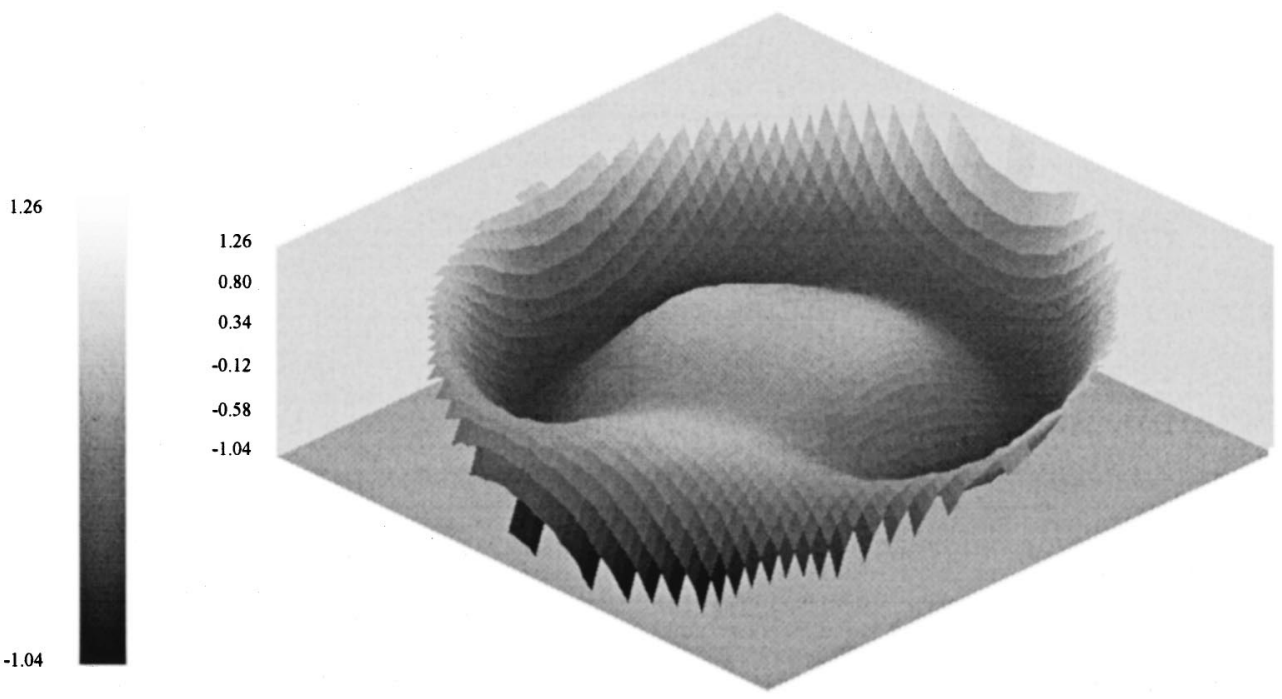

(b)

Fig. 2 (a) Profile of a microlens (350- $\mu$ m lens diameter) before (dashed curve) and after (solid curve) coating with photoresist, measured with a step profiler. (b) Surface deviation from ideal sphere (rms, in units of $\lambda$ ) of a lens after coating (loosely packed array), measured with a Twyman-Green interferometer. 


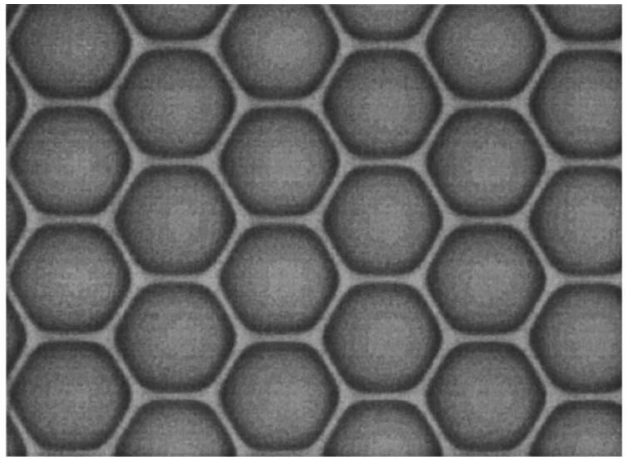

(a)

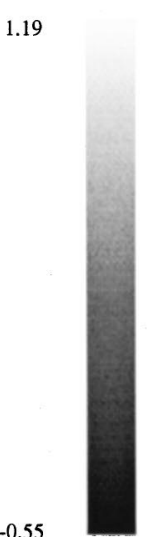

$-0.55$

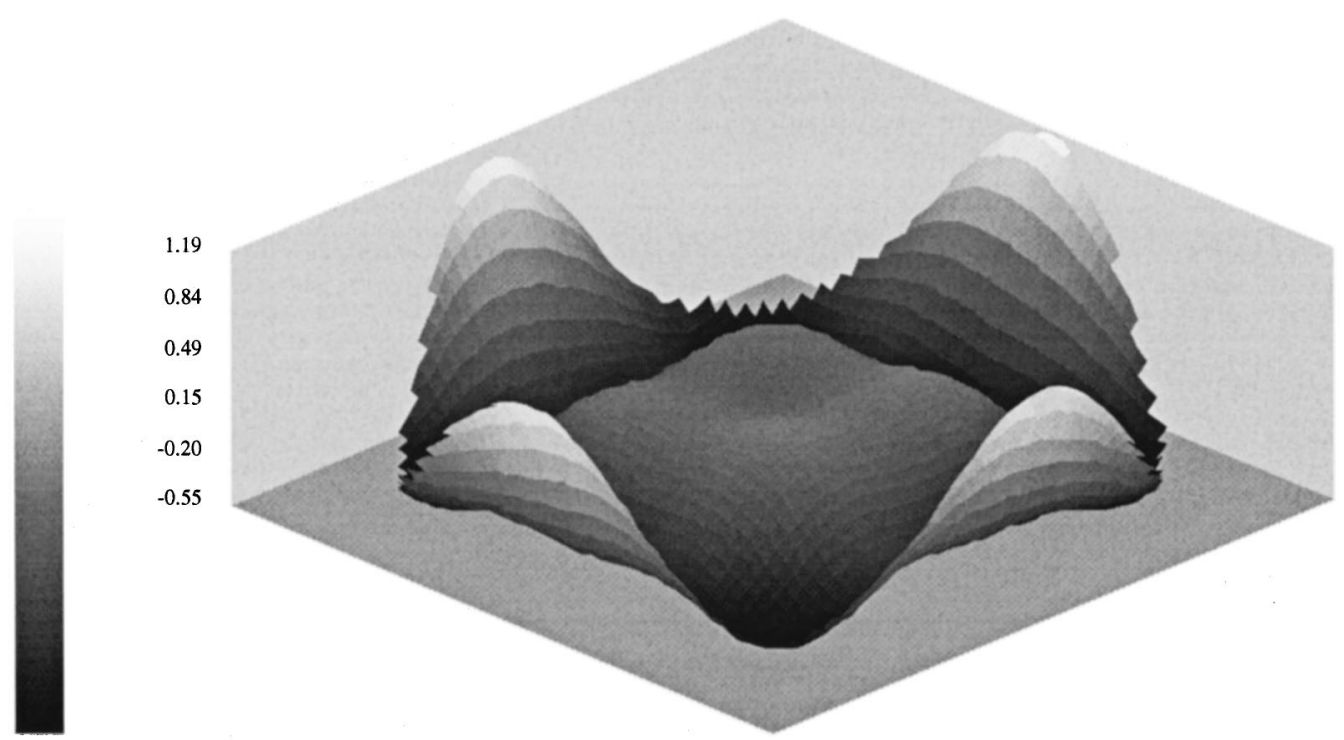

(b)

Fig. 3 (a) Microscope image of a lens array with photoresist coating (hexagonal close-packed). (b) Surface deviation from ideal sphere (rms, in units of $\lambda$ ) of a lens in a close-packed array (square packing) measured with a Twyman-Green interferometer.

We introduce a deviation factor, defined as the deviation measured after spincoating divided by the deviation before coating. The deviation factor is used to quantify the degradation of the original lens surface caused by the spincoating process. For lenses with smaller diameters, the change of profile is much more significant than for the larger ones. This is mainly due to the relative contribution of the outer sections of the lens [Fig. 2(a)]. Besides the parameters of the spincoating procedure itself (the sequences of acceleration, speed, and intermediate pauses), the two main parameters influencing the deviation are the coating thickness of the photoresist and the resist viscosity. To reduce the viscosity, the AZ1518 resist is diluted with AZ1500 thinner. With that and with the reduction of resist thickness, we were able to reduce the deviation factor from a maximum of 5 to below 2 (Fig. 4). Note, however, that a deviation factor of unity does not necessarily mean that the surface is unaltered. The deviation from an ideal sphere as measured with the interferometer is a mean value taken across the entire surface of the microlens. Hence the spincoating may locally increase and also locally reduce the initial deviation from an ideal sphere, without changing the overall rms value. For not perfectly spherical lens surfaces (as in our case) this may thus yield a deviation factor of one. But, for a given array of perfectly spherical microlenses, spincoating will always degrade the sphericity due to the directionality of the process.

\subsection{Holographic Recording}

The choice of holographic lithography for the realization of the diffraction grating was governed by two main advan-

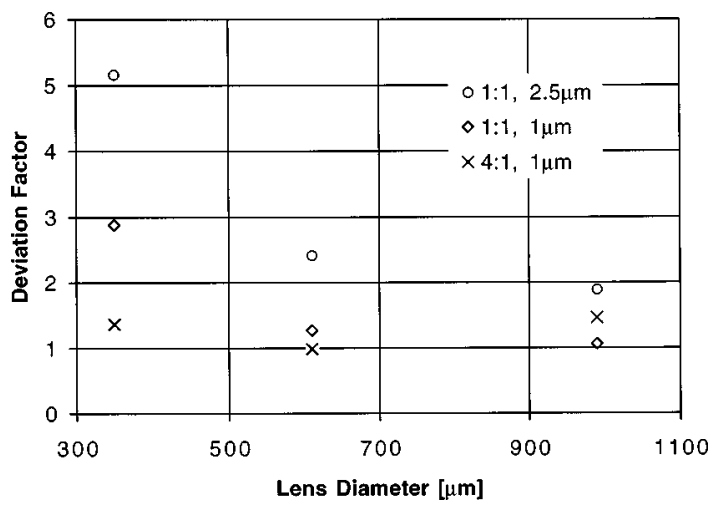

Fig. 4 Change of lens surface quality for different lens diameters. The parameters are resist dilution and deposition thickness. 


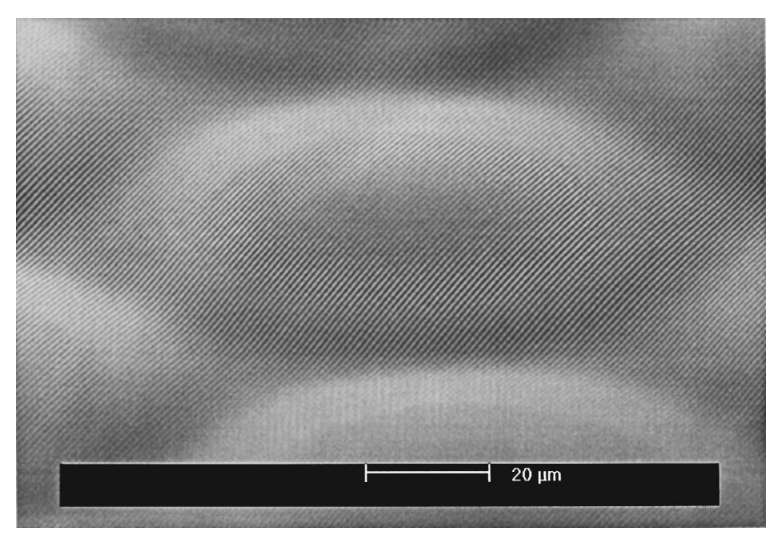

Fig. 5 Electron microscope image of a refractive lens $(100-\mu \mathrm{m}$ diameter) with a diffractive surface (1- $\mu \mathrm{m}$ grating period).

tages of this technology. Besides the fact that a single exposure can structure large areas, the interference is also fairly insensitive to substrates with depth variation. In contrast to conventional mask photolithography, the intensity pattern of two interfering plane waves (which are symmetric with respect to the normal of the substrate) is homogeneous and invariant with depth. This property allows uniform structuring of very fine features on curved surfaces.

Lens arrays with a photoresist coating of $1 \mu \mathrm{m}$ were prebaked at $85^{\circ} \mathrm{C}$ for $40 \mathrm{~min}$. We exposed a grating with a period of $1 \mu \mathrm{m}$ and a depth of about $0.5 \mu \mathrm{m}$ using a $\mathrm{Kr}^{++}$ laser at $413 \mathrm{~nm}$. For further reduction of the coating thickness and hence the spincoating effect, the samples were previously exposed homogeneously with one laser beam before writing the grating. Figure 5 shows an example of a refractive lens with a diffraction grating on the surface.

\section{Characterization}

The fabricated elements were characterized with respect to their performance as spectrometer components in two different spectrometer setups. The two main attributes characterized are stray-light suppression and resolution.

\subsection{Setup with Pinhole}

The first setup is shown in Fig. 6(a). The hybrid element is set-up in a 1:1 imaging system between a $10-\mu \mathrm{m}$ pinhole and a one-dimensional detector array (Hamamatsu, 1024 pixels, $25-\mu \mathrm{m}$ pitch, 16- $\mu \mathrm{m}$ width). The detector is placed parallel with respect to the plane of the hybrid element at a distance of $2 f$ (about $8 \mathrm{~mm}$ ), and the pinhole is aligned on the optical axis of the microlens. Note that the detector pixels are of rectangular shape, which is ideal for a slit, but increases the contribution of stray light for a round pinhole.

The different wavelengths were generated with an $\mathrm{Ar}^{+}$ and two HeNe lasers (543.5 and $632.8 \mathrm{~nm}$ ). For the determination of the resolution, the recorded intensity distributions for the different wavelengths were fitted to a Gaussian function. After the Rayleigh criterion the resolution is defined as the full width at half maximum (FWHM) of the Gaussian function. Figure 6(b) shows the results of the resolution measurements for an element with a diameter of $990 \mu \mathrm{m}$. A maximum resolution of $3 \mathrm{~nm}$ is achieved at the wavelength of $514.5 \mathrm{~nm}$. The resolution then degrades slightly towards the shorter wavelength range and rather

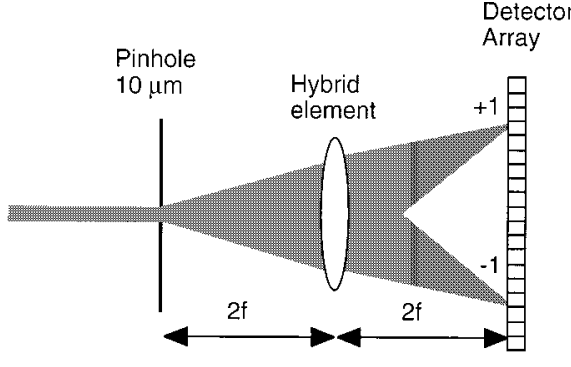

(a)

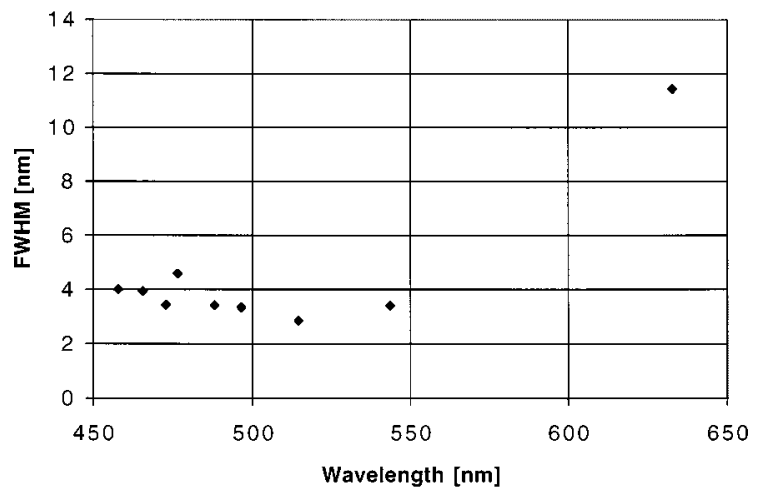

(b)

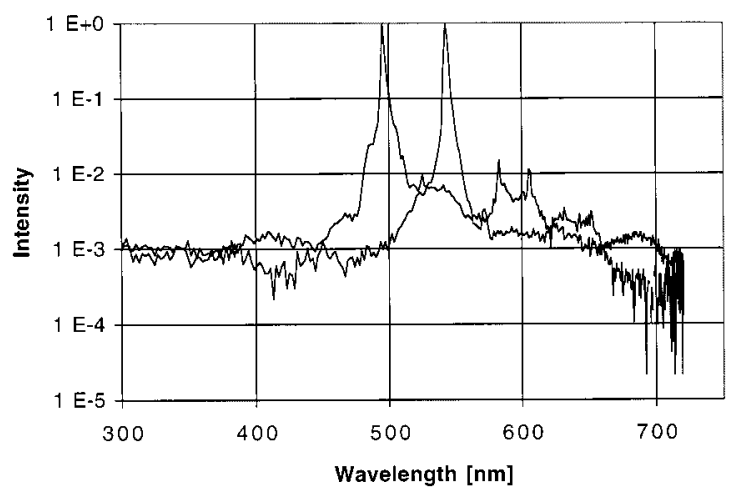

(c)

Fig. 6 (a) Spectrometer setup with a pinhole for the characterization of the hybrid element (990- $\mu$ m lens diameter). (b) Resolution measurements for the different wavelengths of $\mathrm{an}^{+}$and two $\mathrm{HeNe}$ lasers (543.5 and $632.8 \mathrm{~nm}$ ). (c) Stray-light suppression measurements at 496.5 and $543.5 \mathrm{~nm}$.

strongly towards the longer wavelengths. This can be explained by geometrical considerations: the different wavelengths are diffracted at different angles, which in return means that the foci for the different wavelengths do not lie on a straight line but rather on a curve in space (see also raytracing results in Sec. 4). So the shape of the resolutionversus-wavelength curve depends on the position of the image plane in which the detector is placed. In our case, the setup was aligned for maximum resolution at $514.5 \mathrm{~nm}$. For the stray-light suppression measurements we again coupled single laser lines into the same spectrometer setup and analyzed how much intensity is detected on the neighboring pixels. The spectrum is plotted on a logarithmic scale in Fig. 6(c) with the peak value normalized to one. Attenuations of 25 and $30 \mathrm{~dB}$ are measured for the wavelengths 496.5 and $543.5 \mathrm{~nm}$ respectively. 
Traut and Herzig: Holographically recorded gratings on microlenses ...

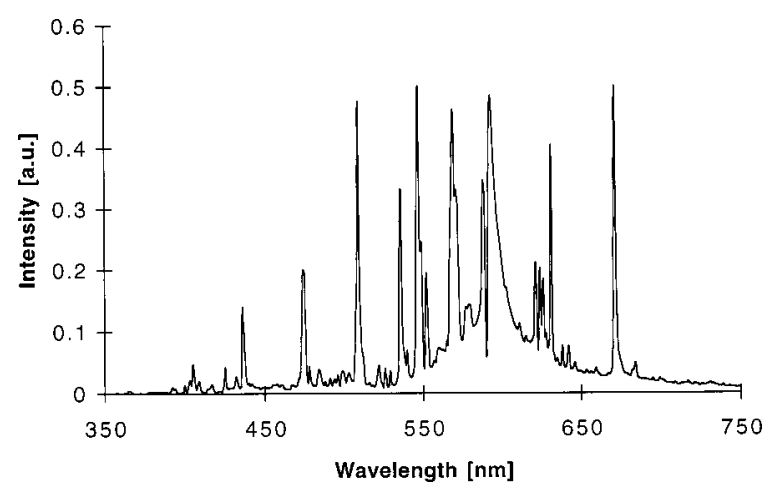

(a)

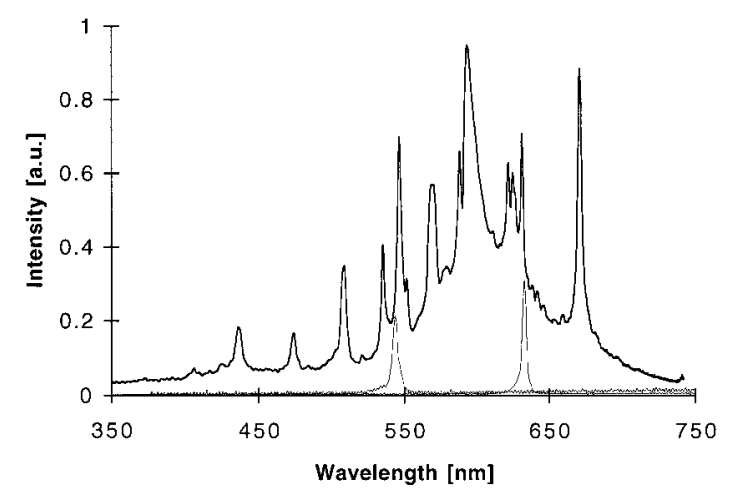

(b)

Fig. 7 Spectrum of a low-pressure xenon lamp measured with (a) a conventional spectrometer HR 250, 1-nm resolution, and (b) a hybrid element. In (b) the two peaks in the separate (gray) plot are the calibration lines from two HeNe lasers (543.5 and $632.8 \mathrm{~nm}$ ).

Figure 7 shows the comparison of a conventional spectrometer (HR250) with a resolution of $1 \mathrm{~nm}$ and one of our microspectrometer elements (lens diameter $990 \mu \mathrm{m}$ ). For test purposes we measured the spectrum of a low-pressure xenon lamp. These two images show the larger amount of stray light for the hybrid element. The resolution in the mid-visible part is better than in the blue-violet and deep red regions, since the detector is placed in the focus for the mid-visible region. Note also that the peaks in the UV range decrease. We attribute this fact to the high absorption of photoresist in the blue-violet wavelength region.

\subsection{Setup with Optical Fiber}

Figure 8(a) shows the second spectrometer setup. Here, the element is set up in a 1:1 imaging system between the exit of an optical fiber (core diameter $8 \mu \mathrm{m}, \mathrm{NA}=0.11$ ) and the same detector array (Hamamatsu) as before. In this case, the single lines were generated by a monochromator. This setup was again adjusted to maximum resolution in the green wavelength range and results in a maximum resolution of $8 \mathrm{~nm}$ at the wavelength of $550 \mathrm{~nm}$. Figure 8(b) shows a strong degradation of the resolution towards both ends of the wavelength spectrum. The stray-light suppression was measured with a filter test. In this test, we measure the transmission spectrum of a white light source (xenon, high pressure, with a uniform spectrum) through an edge filter, which cuts off the spectrum at a particular wavelength. The transmission spectrum is normalized to the uni-

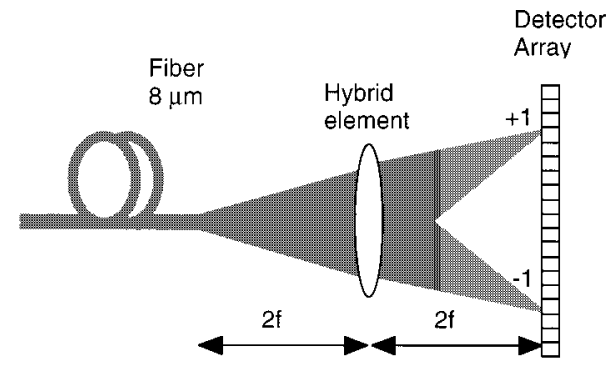

(a)

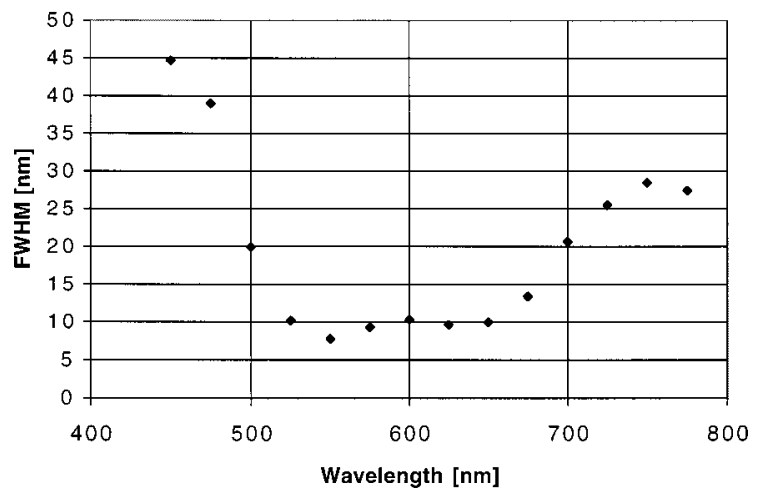

(b)

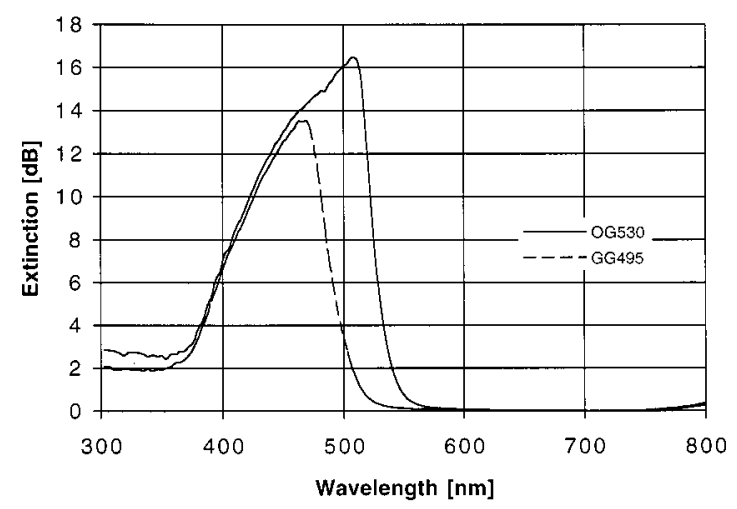

(c)

Fig. 8 (a) Spectrometer setup with an optical fiber for the characterization of the hybrid element $(990-\mu \mathrm{m}$ lens diameter). (b) Resolution measurements for the different wavelengths from the monochromator. (c) Stray-light suppression measurements with two edge filters (cutoffs at 495 and $530 \mathrm{~nm}$ ).

form white light spectrum, and the light still transmitted into the "dark" region is analyzed. Figure 8(c) shows the measurement for a hybrid element with a lens diameter of $990 \mu \mathrm{m}$. The attenuation is measured with the filters GG495 and OG530 (cutoff wavelengths 495 and $530 \mathrm{~nm}$ ) and amounts to 13 and $16 \mathrm{~dB}$, respectively.

The difference in performance for the two spectrometer setups lies in the illumination part. In the first setup, the laser light passes through a pinhole of $10-\mu \mathrm{m}$ diam placed at a distance of $2 f$ (about $8 \mathrm{~mm}$ ) from the element. The circle of illumination on the hybrid element is given by diffraction $(1.2 \mathrm{~mm})$ and matches the diameter of our element quite well. For the setup with the fiber, however, the numerical apertures of the fiber and the lens system are not matched. The fiber placed at a distance of $2 f$ from the 


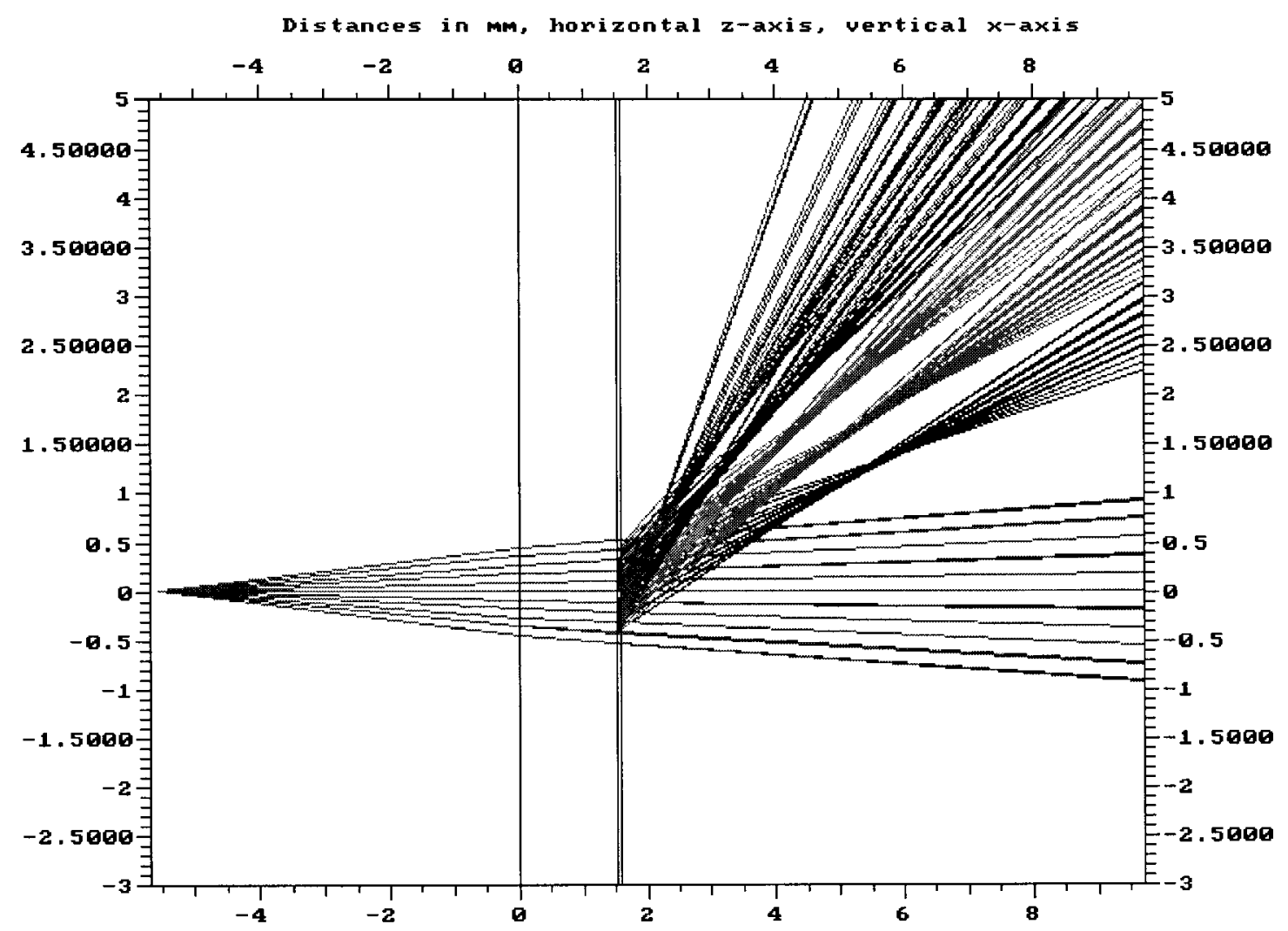

(a)

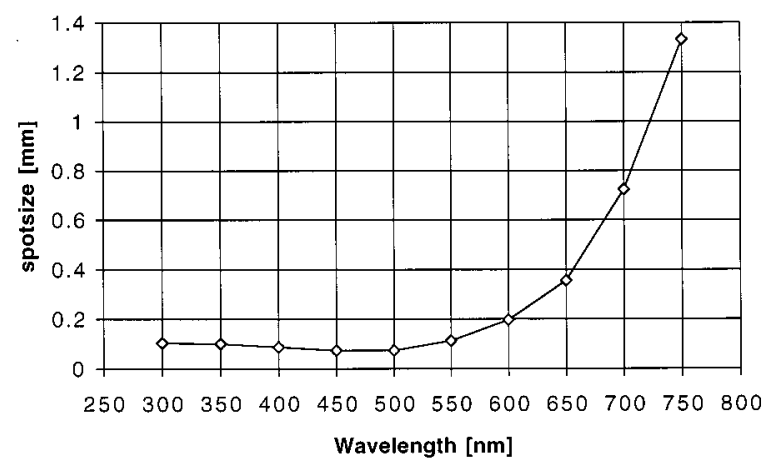

(b)

Fig. 9 Raytracing of a microspectrometer element for normal incidence: (a) Raytracing setup (scale differs for the two axes). (b) Spot sizes (maximum diameter) in the plane of best focus for $500 \mathrm{~nm}$ (parallel to the hybrid element).

element illuminates more than three times the area of the microlens. Since the entire wafer is covered with the grating, we have a contribution of nonfocused diffracted light from the regions between the lenses. For shorter wavelengths, this stray light concentrates around the focus. For longer wavelengths, however, it is distributed over a larger area and at a longer distance from the focal point. The result is a strong degradation of resolution towards the short-wavelength end of the spectrum for this setup, contrary to what was observed for the setup with the pinhole.

To improve the performance, the fiber-lens system has to be matched with respect to numerical aperture or diaphragms have to be introduced between the lenses to block the stray light.

\section{Raytrace Analysis}

For a qualitative analysis of the elements, raytrace simulations were performed with Raytrace 6.2 (N. Lindlein, Lehrstuhl für Optik, University of Erlangen, Germany). The substrate is glass, and the lens is photoresist. The light source is a point source composed of different wavelengths and placed at a distance of $2 f$ from the microlens. We used apertures to create an ideal situation, so there is no contribution of stray light from the regions outside the lens. Two setups were calculated. For the first setup the light source was placed on the optical axis, as for our spectrometer setups. The results are shown in Fig. 9(a), where we traced wavelengths between 300 and $700 \mathrm{~nm}$. As mentioned ear- 


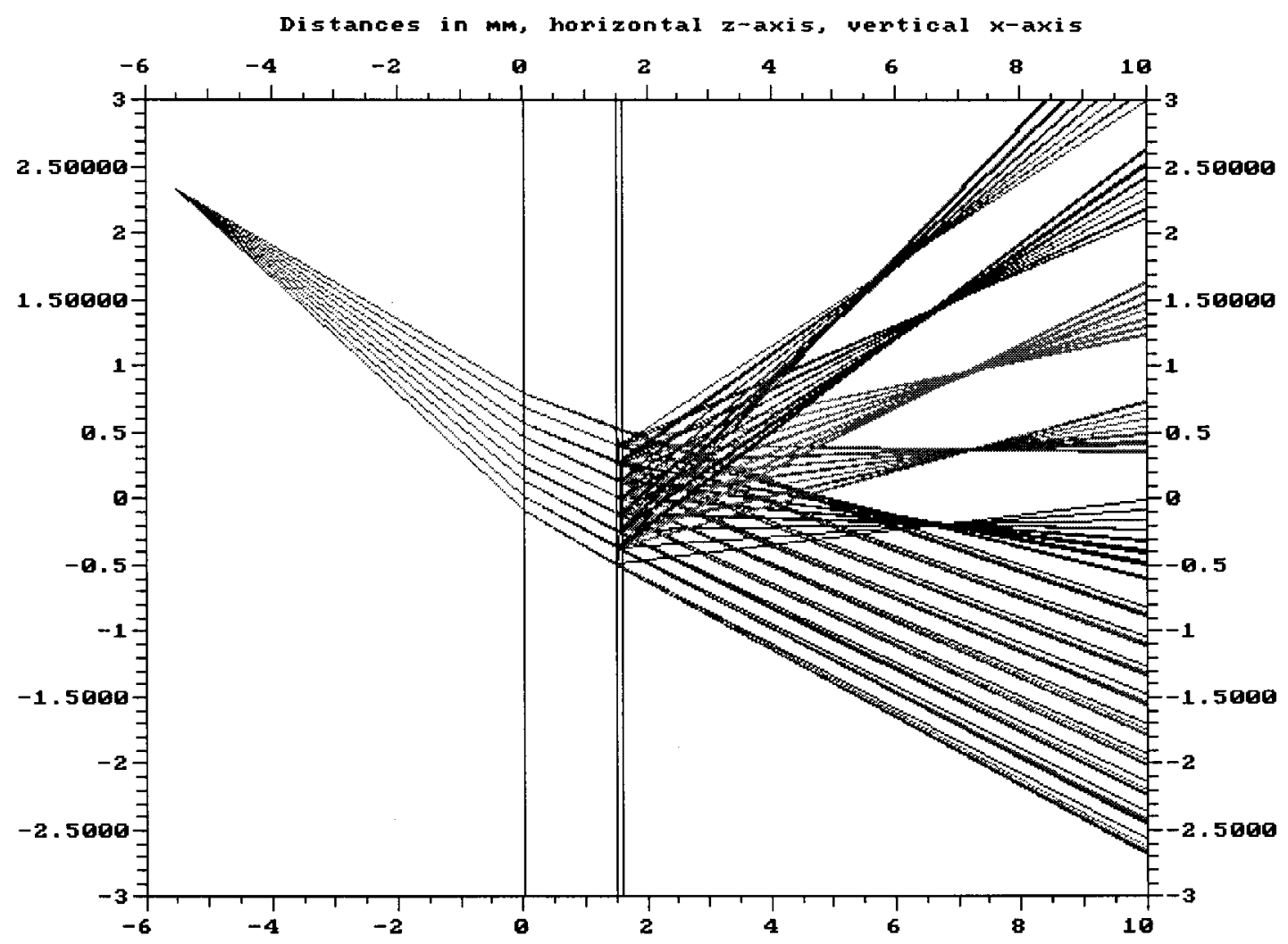

(a)

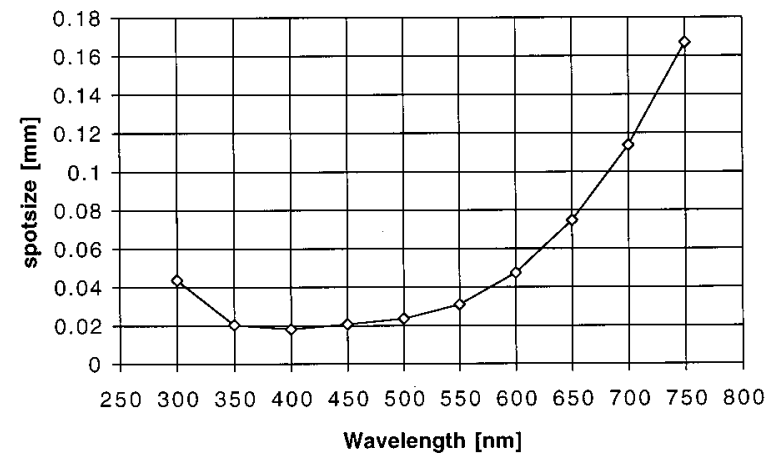

(b)

Fig. 10 Raytracing of a microspectrometer element for oblique incidence: (a) Raytracing setup (scale differs for the two axes). (b) Spot sizes (maximum diameter) in the plane of best focus for $500 \mathrm{~nm}$ (parallel to the hybrid element).

lier, we find that the foci lie on a curve in the $x z$ plane. Figure 9(b) shows the spot sizes calculated for different wavelengths. These spot sizes are calculated in a plane parallel to the substrate to simulate the detector. The detector plane for this calculation was put at the best focus (smallest spot size) for a wavelength of $500 \mathrm{~nm}$. We see that the spot size increases only slightly on going from the minimum at $500 \mathrm{~nm}$ to shorter wavelengths. However, for longer wavelengths, the increase in spot size is much faster. At $750 \mathrm{~nm}$ the spot size is 14 times larger than the minimum value at $500 \mathrm{~nm}$. Recalling the resolution measurements performed in Sec. 3.1, this result corresponds quite well to the resolution measurements presented for the first spectrometer setup [Fig. 6(b)]. It does not, however, match the resolution measurements performed with the second spec- trometer setup, since the stray light from the regions outside the lens is not taken into account in the raytracing calculations.

For the second raytracing setup, we placed the point source off axis to generate rays of oblique incidence on the lens. We see in Fig. 10(a) that the foci still lie on a curve; however, the curvature is much smaller. Looking at the spot sizes calculated in the plane of best focus (minimum spot size) for $500 \mathrm{~nm}$ [Fig. 10(b)], we find two important differences: The spot sizes for this setup are smaller overall, and the increase of the spot size towards the ends of the spectrum is much slower. We therefore suggest using the microspectrometer element with a light source generating rays for oblique incidence. 


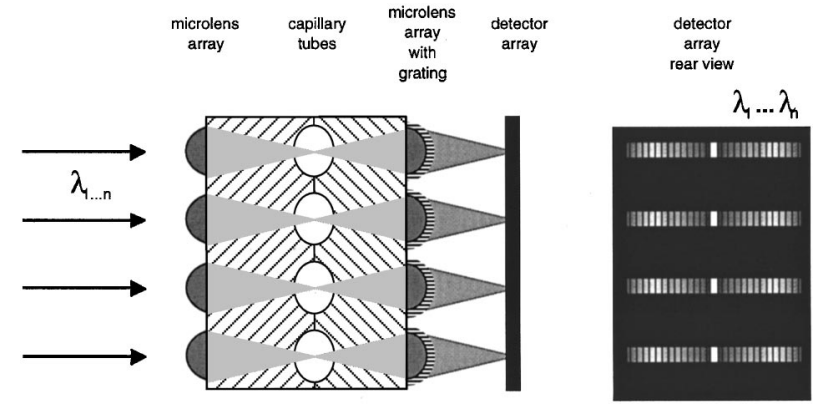

Fig. 11 Proposed concept of a system for a microspectrometer array.

\section{Summary and Conclusion}

We have studied the performance of refractive microlens arrays with diffractive surfaces as elements for miniaturized spectrometer systems. We fabricated arrays of elements that combine two main optical functions, namely focusing and dispersion, on one transmitting surface by mixing different manufacturing technologies used in micro-optics: photolithography and reflow technique for the fabrication of the refractive microlens array, and holography for the diffraction grating on top of the lens array. The main problem in the fabrication process was found to be the photoresist coating for the diffraction grating. The spincoating introduces directionality in the overall fabrication process, which disturbs the otherwise perfect two-dimensional symmetry of the microlens fabrication, which in turn leads to a degradation of the spherical surface of the lens and hence to a deterioration of the lens properties. The result is larger spot sizes and thus lower resolution. To eliminate this problem, a deposition technique should be applied for the photoresist coating that exhibits the same two-dimensional symmetry as the lens fabrication process, such as spraying. A promising alternative is the elimination of the coating step altogether, which could be done if the microlenses were fabricated by "melting", the cylinders in a controlled solvent atmosphere. ${ }^{9}$ For these microlenses, the photoresist remains photosensitive and the grating can be written directly into the lens surface.

With respect to the application of the element in the context of a microspectrometer array, we measured the resolution and the stray-light suppression. Measurements with the spectrometer setup, where the light passes through a pinhole, give a maximum resolution of $3 \mathrm{~nm}$ and a maximum stray-light suppression of $25 \mathrm{~dB}$. The results for the spectrometer setup, that uses a fiber to couple the light into the element were not quite as good $(8 \mathrm{~nm}, 16$ $\mathrm{dB})$. This difference is mainly due to the contribution of nonfocused light diffracted from the regions outside the lens. The following solutions are proposed: either the lensfiber system is matched or diaphragms are utilized with the lenses.

The raytracing results match the experimental results quite well and thus provide good qualitative information, but no quantitative results at this stage. For quantitative information more rigorous theoretical analysis has to be employed and diffraction efficiencies have to be taken into consideration. ${ }^{10}$ Furthermore, the calculations show that better resolution over a larger bandwidth can be expected when operating the element at oblique incidence.

The advantages of the fabricated hybrid element for implementation in a spectrometer array system is obvious on looking at Fig. 11. It presents a schematic view of a possible concept for a microspectrometer array system as used for chemical analysis. The heart of the proposed system consists of two components. The first component implements a microlens array on one side and one half of a capillary tube on the other side. The microlens array focuses the light into the capillary tube, which contains the chemical substance to be analyzed. The second element consists of the other half of the capillary tube on one side and the microlens array with the diffraction grating on top on the other side. These two components contain the capillary tubes and all optical functions necessary. Accordingly, there are only two pieces that are critical in terms of alignment for the entire system, with the exception of the light source. With appropriate replication techniques, ${ }^{11,12}$ the proposed system promises to be very attractive for low- cost applications. The replication of the elements presented is consequently an important issue, which will be investigated in the near future.

\section{Acknowledgments}

The work was supported by the Swiss Priority Program OPTIQUE. The authors would like to thank H. Teichmann and O. Manzardo for valuable discussions and their help with the measurements. Furthermore, the authors gratefully acknowledge the work of I. Philipoussis in the fabrication of the microlens arrays.

\section{References}

1. H. P. Herzig, Ed., Micro-optics Elements, Systems, and Applications, Taylor \& Francis, London (1997)

2. J.-C. Roulet, K. Fluri, E. Verpoorte, R. Völkel, H.-P. Herzig, N. F. de Rooij, and R. Dändliker, "Micro-optical systems for fluorescnce detection in $\mu$ TAS applications," in Micro Total Analysis Systems '98, D. J. Harrison, and A. van den Berg, Eds., pp. 287-290, Kluwer Academic Publishers, Dordrecht (1998).

3. C. Müller and J. Mohr, "A Microspectrometer fabricated by the LIGA process," Interdisciplinary Sci. Rev. 18, 273-279 (1993).

4. E.-B. Kley and B. Schnabel, "E-beam lithography: a suitable technology for fabrication of high accuracy 2D and 3D surface profiles," in Micromachining and Microfabrication '95, Proc. SPIE 2640, 71-80 (1995).

5. F. Sauer, J. Jahns, C. R. Nijander, A. Y. Feldblum, and W. P. Townsend, "Refractive-diffractive micro-optics for permutation interconnects," Opt. Eng. 33, 1550-1560 (1994).

6. A. Schilling, P. Nussbaum, C. Ossmann, S. Traut, M. Rossi, H. Schift, and H. P. Herzig, "Miniaturized focusing fan-out elements: design, fabrication and characterization," J. Opt. A: Pure Appl. Opt. 1, 244248 (1999)

7. M. C. Hutley, "Refractive lenslet arrays," in Ref. 1, pp. 127-152.

8. J. Schwider and O. Falkenstörfer, "Twyman-Green interferometer for testing microspheres," Opt. Eng. 34, 2972-2974 (1995).

9. L. Erdmann and D. Efferenn, "Technique for monolithic fabrication of silicon microlenses with selectable rim angles,"' Opt. Eng. 36, 1094-1098 (1997)

10. M. A. Golub and I. Grossinger, "Analysis and modeling of nonparaxial diffractive optical elements on curvilinear surfaces," in Diffractive Optics and Micro-optics, Vol. 10, pp. 75-77, OSA Technical Digest Series, Optical Society of America, Washington (1998).

11. M. T. Gale, "Replication technology for holograms and diffractive optical elements," J. Imaging Sci. Technol. 41, 211-220 (1997).

12. M. T. Gale, "Replication," in Ref. 1, pp. 153-177. 


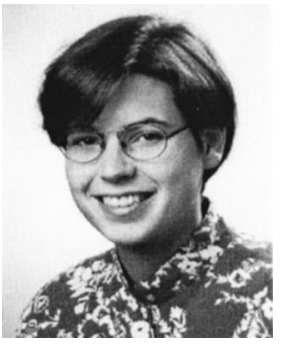

S. Traut received her BS in engineering physics from the Fachhochschule Lübeck and her MS in applied physics from the University of Massachusetts in 1997. Her studies in the USA were made possible with a scholarship from the Fulbright Commission. For her diploma work, she was engaged by IBM Research in Rueschlikon, Switzerland, to work in the process development of GaAs-based lasers. Her master's thesis at CEMSOS, UMass Lowell, involved work on photorefractive $\mathrm{BaTiO}_{3}$. Currently she is working as a research assistant with the Institute of Microtechnology at the University of Neuchtel on the fabrication and characterization of microoptical elements.

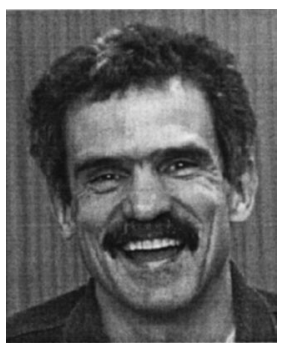

H. P. Herzig received his diploma in physics from the Swiss Federal Institute of Technology in Zürich, Switzerland, in 1978. From 1978 to 1982 he was a scientist with the Optics Development Department of Kern in Aarau, Switzerland, working in lens design and optical testing. In 1983, he became a graduate research assistant with the Applied Optics Group at the Institute of Microtechnology of the University of Neuchâtel, Switzerland, working in the field of holographic optical elements, especially scanning elements. In
1987, he received his PhD degree in optics. He currently heads the micro-optics research group and is an associate professor at the University of Neuchâtel. Dr. Herzig is the member of OSA and EOS and a board member of the Swiss Society of Optics and Microscopy. 